\title{
Protective Effect of Metformin on Doxorubicin-induced Cardiomyopathy in the Adult Male Albino Rats (Light and Electron Microscopic Study).
}

Ahmed A Agaba ${ }^{1 *}$, MSc; Mohamed M Ebada ${ }^{1}, \mathrm{PhD}$ and Hussein M Emara ${ }^{1}, \mathrm{PhD}$

\author{
* Corresponding Author: \\ Ahmed Ahmed Agaba \\ ah_agaba@yahoo.com
}

Received for publication April 18, 2021; accepted April 26, 2021; published online May 1, 2021

Copyright 2021 The Authors published by Al-Azhar University, Faculty of Medicine, Cairo, Egypt. All rights reserved. This an openaccess article distributed under the legal terms, where it is permissible to download and share the work provided it is properly cited. The work cannot be changed in any way or used commercially.

doi: 10.21608/aimj.2021.72417.1458

${ }^{1}$ Department of Anatomy and Embryology, Faculty of Medicine Al-Azhar University.

\begin{abstract}
Background: Cancer is considered as the leading cause of death in the majority of countries all over the world. Doxorubicin belongs to anthracycline group and is widely used in cancer treatment, but cardiotoxicity is one of its serious adverse effects. Metformin, a wellknown anti-diabetic drug, has cardioprotective effects and thought to protect against doxorubicin-induced cardiotoxicity.

Objective: to reveal the histological and biochemical alterations in the myocardium of rats treated with doxorubicin, and to explore the impact of metformin co-treatment.

Material and methods: The study was done at the Animal House, Faculty of Pharmacy, Al-Azhar University between July and September 2020. Sixty adult male albino rats were used (equally divided into 5 groups); group I: given $1 \mathrm{ml}$ normal saline orally every day, and also injected intraperitoneally by $1 \mathrm{ml}$ saline every other day for 12 days; group II: given $250 \mathrm{mg} / \mathrm{kg} /$ day of metformin orally for 12 days; group III: given $3 \mathrm{mg} / \mathrm{kg}$ of doxorubicin intraperitoneally every other day for 12 days; group IV: given $250 \mathrm{mg} / \mathrm{kg} /$ day of metformin orally and 3 $\mathrm{mg} / \mathrm{kg}$ of doxorubicin intraperitoneally every other day for 12 days; group V: given $3 \mathrm{mg} / \mathrm{kg}$ of doxorubicin intraperitoneally every other day for 12 days, then left 2 months for observation from the last dose. Then, the animals were anesthetized and tissues of heart were proceeded and microscopically examined. Also, blood samples were taken for biochemical investigation.

Results: In doxorubicin group, there were severe histological changes and biochemical results showed highly elevated cardiac enzymes. These results were significantly improved in metformin co-treatment and minimally improved in withdrawal group.

Conclusion: This study revealed that metformin might have a protective effect against doxorubicin-induced cardiotoxicity.

Keywords: Metformin; doxorubicin; cardiotoxicity; myocardium; rats.

Authorship: All authors have a substantial contribution to the article.

Disclosure: The authors have no financial interest to declare in relation to the content of this article. The Article Processing Charge was paid for by the authors.
\end{abstract}

\section{INTRODUCTION}

Cancer affects most populations worldwide. It is recorded as the second leading cause of mortality in $2018^{1}$. Chemotherapy is an important type of cancer treatment together with radiotherapy and surgery, but its cardiotoxicity leading to cardiomyopathy remains a major side effect ${ }^{2}$.

Cardiomyopathy -in general- means decreased pumping power of the heart, and hence, can lead to heart failure. It is the third cause of heart failure after coronary insufficiency and hypertension ${ }^{3}$.

Doxorubicin (DOX) is an anthracycline chemotherapy which can be used alone or together with other medical treatments to help in cancer treatment, including acute leukemia, malignant lymphoma, non-Hodgkin's and Hodgkin's lymphoma, and other types of cancer ${ }^{4}$.
Like other chemotherapeutic agents, DOX has serious cardiac side effects including cardiotoxicity. DOX-induced cardiotoxicity includes cardiomyocyte apoptosis and myocardial fibrosis leading eventually to heart failure unresponsive to medical treatment ${ }^{5}$. The proposed cardiotoxic mechanisms of DOX include myocardial injury induced by free radicals, mitochondrial damage, and cellular toxicity ${ }^{6}$. The role of reactive oxygen species (ROS) in DOX cardiotoxicity is enhanced by the cardioprotective effect of antioxidants in animals treated with $\mathrm{DOX}^{7}$. Clinical trials over the past years revealed that there are many compounds have a role in preventing or reducing DOX severe cardiotoxicity and also, enhancing its antitumor activity. Therefore, oncologists can use DOX in clinical situations safely 
${ }^{8}$. Metformin (MET) is one of these compounds which protect against DOX cardiotoxicity ${ }^{9}$.

MET is an oral drug used as a first line treatment in type II diabetes mellitus management ${ }^{10}$. Besides its anti-diabetic effects, MET has antioxidant effect and also decreases the lipid peroxidation ${ }^{11}$.

Moreover, a recent study on diabetic patients has shown that, patients using MET have a lower risk for developing cancer compared to the patients using other anti-diabetic medications including insulin ${ }^{12}$. Therefore, this study aimed to reveal DOX effect on the heart and its ability to induce cardiomyopathy in the adult male albino rats. This study also tried to explore the effect of MET in the prevention and treatment of doxorubicin-induced cardiomyopathy.

\section{MATERIAL AND METHODS}

Animals:

Sixty adult male albino rats aging $12-14$ weeks and weighing 200-230 grams were used. The study was done at Animal House, Faculty of Pharmacy, AlAzhar University, Cairo, between July and September 2020.

Experimental Design:

The rats were randomly divided into five groups (twelve rats in each group):

Group I (control group): rats were given $1 \mathrm{ml}$ normal saline orally every day by gastric gavage for 12 days, and were also injected intraperitoneally (IP) by $1 \mathrm{ml}$ saline every other day for 12 days ${ }^{11,13,14}$.

Group II (metformin-treated group): rats were given metformin $(250 \mathrm{mg} / \mathrm{kg} /$ day) by a gastric gavage for 12 days, and were also given $1 \mathrm{ml}$ saline IP every other day for 12 days ${ }^{11,13,14}$.

Group III (doxorubicin-treated group): rats were injected IP by DOX (3 mg/kg) every other day for 12 days (the cumulative 6 doses were $18 \mathrm{mg} / \mathrm{kg}$ ) ${ }^{11,13,14}$. Group IV (doxorubicin- and metformin-treated group): rats were given metformin $(250 \mathrm{mg} / \mathrm{kg} /$ day $)$ by gastric gavage for 12 days and were also given DOX IP (3 mg/kg) every other day for 12 days $11,13,14$.

Group V (withdrawal group): rats were injected IP by DOX (3 mg/kg) every other day for 12 days, then rats were left 2 months for observation from the last dose.

Chemicals:

The chemicals used in this work were:

-DOX 50mg vials containing $25 \mathrm{ml}$ red solution, were purchased from Pfizer Egypt Pharmaceutical Company, and given directly without dilution.

-MET 500mg tablets, were purchased from Merck Egypt Pharmaceutical Company, and was given after dissolution of the tablet in $5 \mathrm{ml}$ normal saline.

Methods:

1) General examination of the heart and mortality recording:

The external appearance of the heart was examined by naked eye. Also, the mortality rate was recoded in each group.

2) Measurements (body weight (BW), heart weight (HW), and HW/BW ratio):
BW of each rat was recorded at the $1^{\text {st }}, 6^{\text {th }}$ and $12^{\text {th }}$ days. Also, BW of each rat in the withdrawal group was recorded at the $72^{\text {nd }}$ day. The weight of the heart of each rat was recorded just after sacrificing and heart extraction. Also, the heart/BW ratio was also estimated.

3) Collection of blood samples:

At the $12^{\text {th }}$ day of the experiment, blood samples were withdrawn from all groups, except the withdrawal group samples were withdrawn at the $72^{\text {nd }}$ day. Samples were collected from the rat tail in tubes, centrifuged for $15 \mathrm{~min}$ and then, processed for biochemical assays.

4) Biochemical assay of cardiotoxicity:

Estimation of serum creatine kinase-MB (CK-MB) and serum troponin I (cTn-I) activities were determined using ELISA kits.

5) Heart specimen collection:

The rats in all groups were collected by the end of the $12^{\text {th }}$ day, except in withdrawal group, were collected by the end of the $72^{\text {nd }}$ day. The rats were anaesthetized using ether inhalation. Then, the heart of each rat was dissected out, examined by naked eye, and the great vessels were gently washed by normal saline ${ }^{11,13,14}$.

6) Light and electron microscopic processing:

The collected hearts were divided transversely, and 2 $\mathrm{mm}$ thick section of each heart was immersed in $2.5 \%$ glutaraldehyde solution. Then, they fixed for 2 hours in $1 \%$ osmium tetraoxide at $4^{\circ} \mathrm{C}$. Then after washing the tissue three times in distilled water, the specimens were prepared for dehydration, infiltration, ultramicrotomy. After that, tissues were stained by uranyl acetate and lead citrate for transmission electron microscopic processing (TEM). Then, the rest of each dissected heart was preserved in $10 \%$ buffered formalin and then processed for paraffin sections then stained by hematoxylin and eosin (H\&E) and also Masson's trichrome stain for light microscopic examination ${ }^{11,15}$.

7) Statistical analysis:

Data was evaluated with SPSS 23 using ANOVA test followed by least significant difference (LSD) post hoc test. Data was interpreted as mean \pm standard deviation (SD). Values are considered significant when $\mathrm{P}<0.05$, while values are considered highly significant when $\mathrm{P}<0.001$. Values are considered non-significant when $\mathrm{P}>0.05$.

\section{RESULTS}

1) External appearance and mortality:

Examination of the external appearance of the hearts revealed that there was no difference between the hearts excised from all groups.

Regarding mortality, the control and metformintreated groups showed no death. There was a low mortality rate in combined DOX and metformintreated group ( $8 \%$ mortality rate as one rat died at the 4th day); while a high mortality rate was observed in the DOX-treated and withdrawal groups; $(42 \%$ in the DOX-treated group and 58\% in the withdrawal group as 5 and 7 rats died in each group respectively) (Fig. $1)$. 


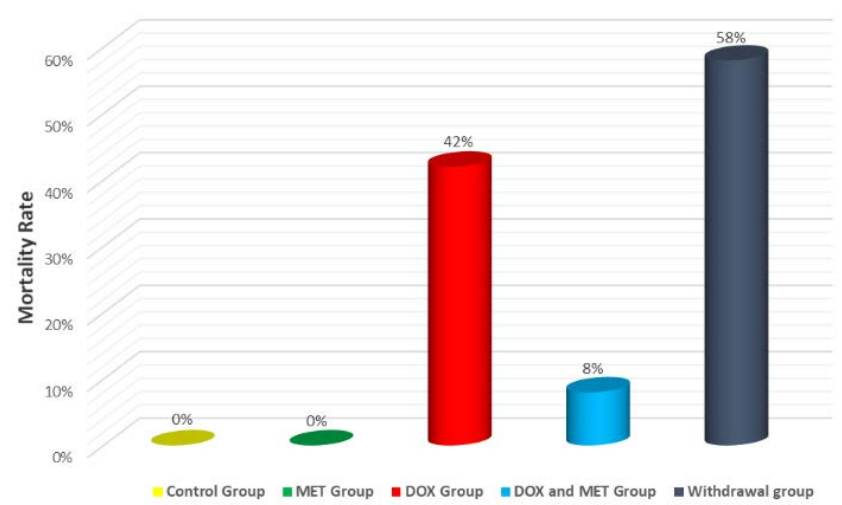

Fig.1: Comparison between all groups regarding mortality rate

2) Body weight, heart weight and heart to body weight ratio:

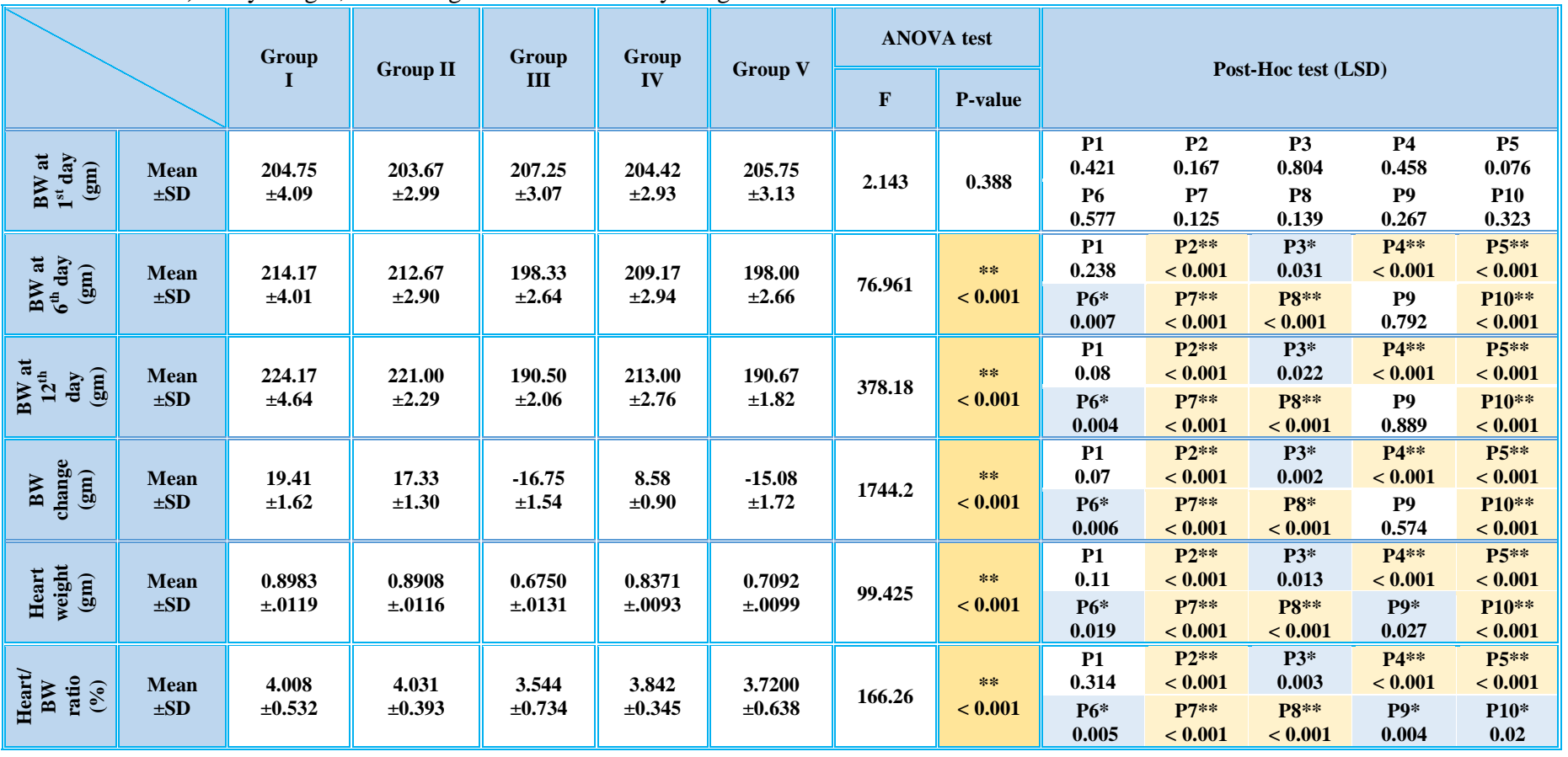

Table 1: Effect of DOX and/or metformin on body and heart weight.

$\mathrm{BW}=$ body weight. $\quad \mathrm{SD}=$ standard deviation. $\quad \mathrm{F}=\mathrm{ANOVA}$ test. $\quad \mathrm{LSD}=$ least significant difference.

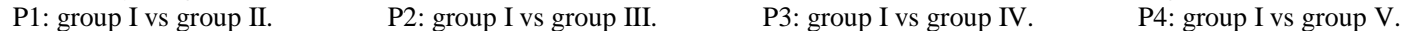

P5: group II vs group III. P6: group II vs group IV. P7: group II vs group V. P8: group III vs group IV.

P9: group III vs group V. P10: group IV vs group V. ** highly significant $\quad$ * significant.

3) Results of cardiac biochemical markers:

\begin{tabular}{|c|c|c|c|c|c|c|c|c|c|c|c|c|c|}
\hline \multicolumn{2}{|c|}{ Variables } & \multirow{2}{*}{$\begin{array}{c}\text { Group } \\
\text { I } \\
\\
\\
\mathbf{0 . 0 1 5 9} \\
\pm \mathbf{0 . 0 1 0 7}\end{array}$} & \multirow{2}{*}{$\begin{array}{c}\text { Group II } \\
\\
\begin{array}{c}0.0158 \\
\pm 0.0108\end{array}\end{array}$} & \multirow{2}{*}{$\begin{array}{c}\text { Group } \\
\text { III } \\
\\
\\
1.0075 \\
\pm 0.1562\end{array}$} & \multirow{2}{*}{$\begin{array}{c}\text { Group } \\
\text { IV } \\
\\
\\
\mathbf{0 . 0 8 8 3} \\
\pm \mathbf{0 . 0 2 4 3}\end{array}$} & \multirow{2}{*}{$\begin{array}{c}\text { Group V } \\
\\
\\
\mathbf{0 . 3 7 6 7} \\
\pm 0.0603\end{array}$} & \multicolumn{2}{|c|}{ ANOVA test } & \multicolumn{5}{|c|}{ Post-Hoc test (LSD) } \\
\hline 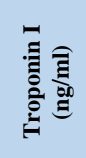 & $\begin{array}{c}\text { Mean } \\
\pm \text { SD }\end{array}$ & & & & & & 444.42 & $\begin{array}{c}* * \\
<0.001\end{array}$ & $\begin{array}{c}\text { P1 } \\
0.998 \\
\text { P6** } \\
<0.001\end{array}$ & $\begin{array}{c}\text { P2** } \\
<0.001 \\
\text { P7** } \\
<0.001\end{array}$ & $\begin{array}{c}\text { P3** } \\
<0.001 \\
\text { P8** } \\
<0.001\end{array}$ & $\begin{array}{c}\text { P4** } \\
<0.001 \\
\text { P9** } \\
<0.001\end{array}$ & $\begin{array}{c}\text { P5** } \\
<0.001 \\
\\
\text { P10* } \\
0.009\end{array}$ \\
\hline 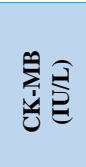 & $\begin{array}{c}\text { Mean } \\
\pm \text { SD }\end{array}$ & $\begin{array}{c}14.3333 \pm \\
3.8217\end{array}$ & $\begin{array}{c}14.5000 \pm \\
4.5025\end{array}$ & $\begin{array}{c}92.1667 \pm \\
8.6951\end{array}$ & $\begin{array}{c}29.5000 \pm \\
3.2333\end{array}$ & $\begin{array}{l}38.6780 \\
\pm 5.3748\end{array}$ & 466.94 & $\begin{array}{c}* * \\
<0.001\end{array}$ & $\begin{array}{c}\text { P1 } \\
0.949 \\
\\
\text { P6** } \\
<0.001\end{array}$ & $\begin{array}{c}\text { P2** } \\
<0.001 \\
\\
\text { P7** } \\
<0.001\end{array}$ & 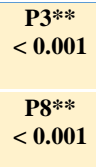 & $\begin{array}{c}\text { P4** } \\
<0.001 \\
\\
\text { P9** } \\
<0.001\end{array}$ & $\begin{array}{c}\text { P5** } \\
<0.001\end{array}$ \\
\hline
\end{tabular}

Table 2: Effect of DOX and/or metformin on cardiac markers. $\mathrm{SD}=$ standard deviation. $\quad \mathrm{F}=$ ANOVA test.

P1: group I vs group II. $\quad$ P2: group I vs group III.

P5: group II vs group III. $\quad$ P6: group II vs group IV.

P9: group III vs group V. $\quad$ P10: group IV vs group V.
LSD= least significant difference.

P3: group I vs group IV. $\quad$ P4: group I vs group V.

P7: group II vs group V. P8: group III vs group IV.

** highly significant. $*$ Significant. 
4) Effect of DOX on myocardial histology:

A. Light microscopic results:

1. Group I (control group) and group II (MET-treated group):

H\&E sections from the heart of the control and MET-treated groups showed similar histologic findings. The cardiac muscle fibers in both groups showed regular arrangement in which the fibers were running almost longitudinally with branching and anastomosing ends. The cardiomyocytes exhibited acidophilic sarcoplasm with visible cross striations and oval vesicular centrally located nuclei (Figs. 2-A and 2-B).

Masson's trichrome sections showed normal collagen distribution in the myocardium. There were rounded thick-walled blood vessels with a mildly thick collagen layer around them. There was also a very thin collagen layer -or nearly absent- in between the muscle fibers (Figs. 3-A and 3-B).

2. Group III (DOX-treated group):

H\&E sections from the heart of DOX-treated group showed disorganized, disrupted and widely separated
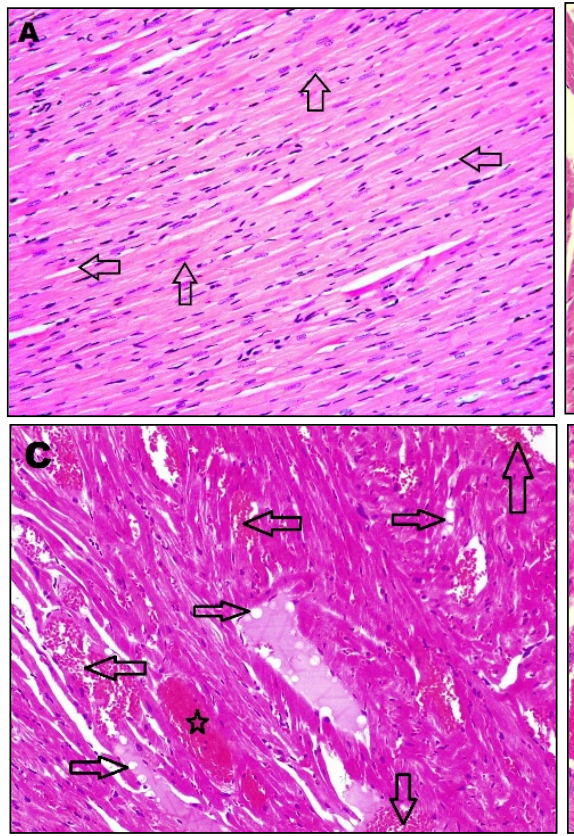

degenerated cardiac muscle fibers. Most fibers exhibited homogenous acidophilic sarcoplasm with pyknotic nuclei. Areas of totally disorganized myofibrils with lost striations, hemorrhage and sarcoplasmic vacuolations are also noticed (Fig. 2C).

Masson's trichrome sections showed thick irregular collagen bundles in between cardiac muscle fibers (Fig. 3-C)

3. Group IV (combined MET- and DOX-treated group):

H\&E sections from the heart of combined MET- and DOX-treated group showed majority of the muscle fibers restored the normal histological pattern with oval, vesicular and centrally located nuclei. No remarkable myofibrillar cells loss was observed, but occasional cardiac muscle fibers with homogenous acidophilic sarcoplasm were detected (Fig. 2-D).

Masson's trichrome sections showed markedly decreased collagen fibers in between cardiomyocytes nearly like collagen distribution of groups I and II (Fig. 3-D).
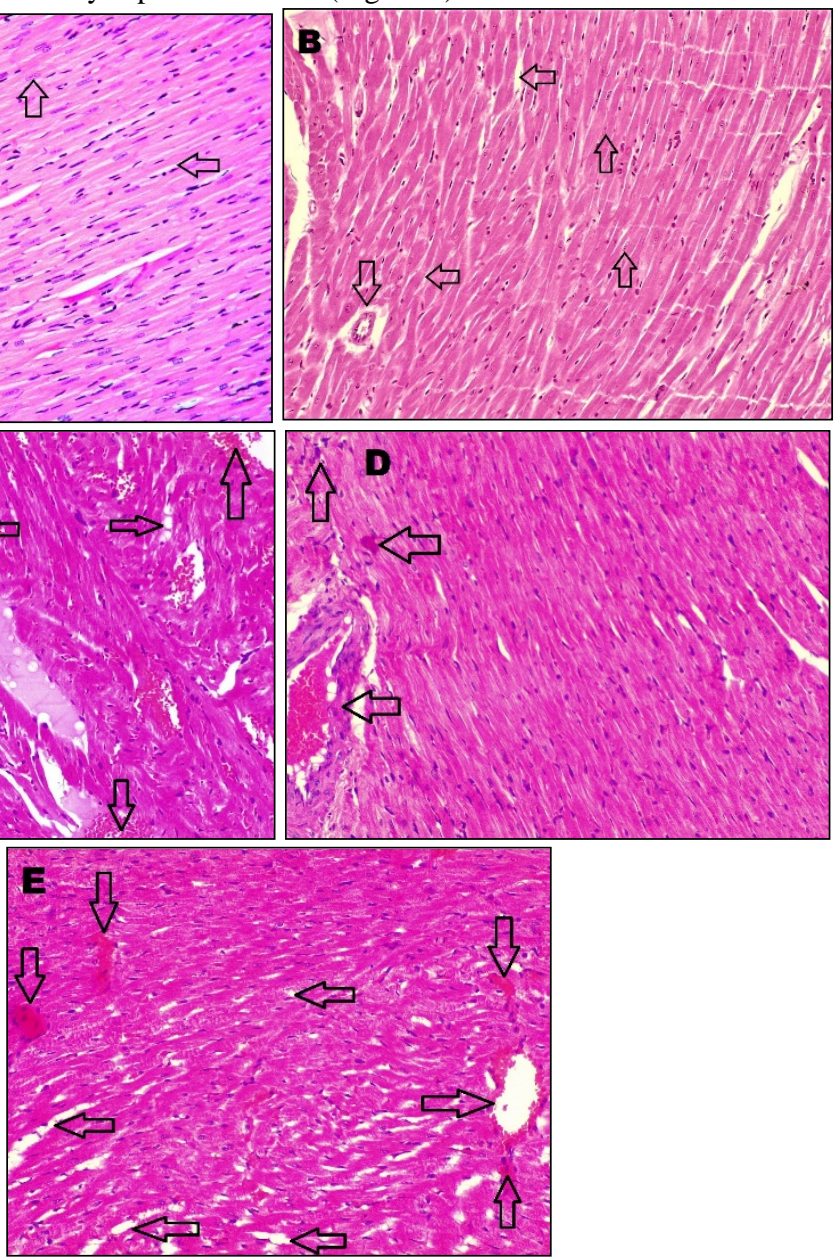

Fig. 2: A photomicrograph of a section of the heart of A) control and B) MET groups showing viable cardiac muscle fibers with centrally located nuclei (up directed arrows), well-distinct cell borders, narrow interstitial space (left directed arrows) and a normal blood vessel (down directed arrow). C) DOX group showing disorganized and degenerated cardiac muscle fibers, areas of hemorrhage (up and down directed arrows), interstitial congestion (left directed arrows) and sarcoplasmic vacuolations (right directed arrows). A large area of myofibrillar degeneration with homogenous hypereosinophilic sarcoplasm is also noted (star). D) Combined DOX and MET group showing normal myocardial fibers with a small area of cardiomyocyte degeneration (upper left directed arrow), dilated 
congested blood vessels (lower left directed arrow) and few inflammatory cellular infiltrations (up directed arrow). E) Withdrawal group showing disrupted and widely separated degenerated cardiac muscle fibers with loss of cross striations, myofibrillar loss (right directed arrow), myofibrillar degeneration (down directed arrows) and sarcoplasmic vacuolations (left directed arrows).

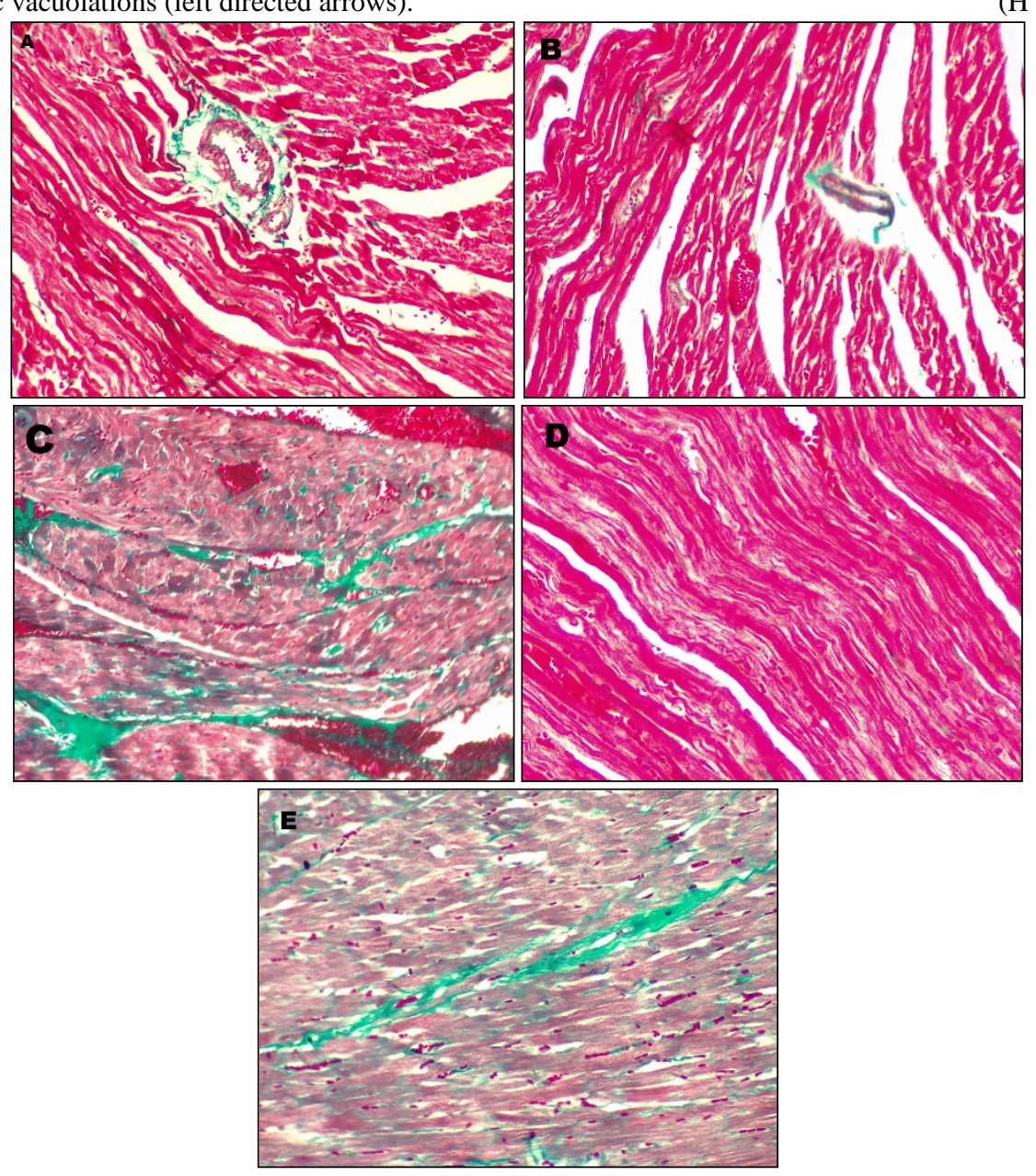

(H \& E, X 200)

Fig. 3: A photomicrograph of a section of the heart of A) Control, B) MET, and D) Combined DOX and MET groups showing normal collagen distribution. C) DOX, and E) Withdrawal groups showing multiple areas of fibrosis with thick irregular collagen layer in between cardiac muscle fibers but more severe in DOX group.

(Masson's trichrome, X 400)

4. Group V (withdrawal group):

H\&E sections from the heart of withdrawal group showed a picture of myocardial damage induced by DOX like the DOX-treated group, but less severe

(Fig. 2-E).

Masson's trichrome sections showed a picture of myocardial fibrosis like the DOX-treated group, but less severe (Fig 3-E).
B. Transmission electron microscopic (TEM) results: 1. Group I (control group) and group II (MET-treated group):

TEM examination of the heart of the control and metformin groups showed similar results in which cardiac myofibrils appeared formed of alternating A and I bands with regular $\mathrm{Z}$ lines. Nuclei of the cardiac muscle fibers were euchromatic with evenly dispersed nuclear chromatin and distinct nucleoli. Mitochondria with closely packed cristae were shown in rows between the cardiac myofibrils and at the poles of nuclei (Figs. 4-A and 4-B). 

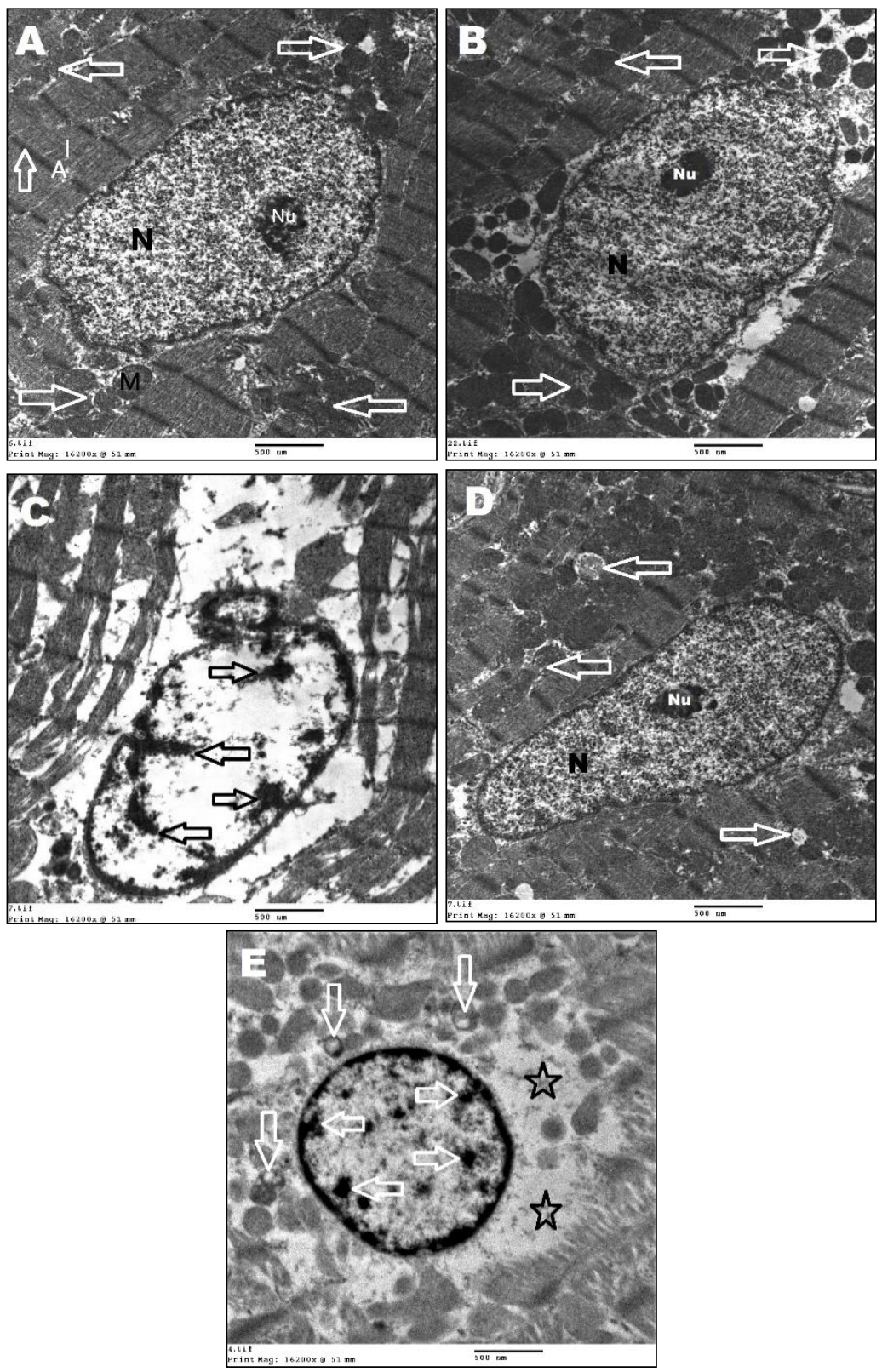

Fig. 4: An electron photomicrograph of sections of the heart of A) Control, and B) MET groups, showing normal myofibrillar structure (up directed arrow), euchromatic oval centrally-located nucleus $(\mathrm{N})$, distinct nucleolus $(\mathrm{Nu})$, mitochondria (M) at the poles of the nucleus (right directed arrows) and in between myofibrils (left directed arrows). C) DOX, and E) withdrawal groups, showing nuclear karyorrhexis (right and left directed arrows) with wide areas of chromatin loss and wide perinuclear area (stars). There is absence of mitochondria in DOX group and most of mitochondria in withdrawal group show disrupted vacuolated cristae (down directed arrows). D) Combined DOX and MET group showing normal architecture with few mitochondria show vacuolated disrupted cristae (right directed arrows) and other show fragmented deficient mitochondrial membrane (left directed arrow).

(TEM, X 15000)

2. Group III (DOX-treated group):

TEM examination of the heart of the DOX-treated group showed focal areas of myofibrillar loss with disrupted sarcomere. The majority of nuclei were pyknotic with aggregated chromatin in the form of electron-dense clumps seen in some parts of the nuclei with wide areas of chromatin loss in other parts. Karyorrhexis was observed in some nuclei. Mitochondria were nearly absent around the pyknotic nuclei, while others appeared fragmented with disrupted cristae (Fig. 4-C).

3. Group IV (combined MET- and DOX-treated group):

TEM examination of the heart of the combined MET- and DOX-treated group showed architecture similar to control group with minor loss in limited focal areas. Most nuclei of cardiac muscle fibers showed normal chromatin. The majority of the 
mitochondria showed closely packed cristae, while few showed disrupted cristae (Fig. 4-D).

4. Group V (withdrawal group):

TEM examination of the heart of the withdrawal group showed a picture of myocardial damage like the DOX-treated group, but less severe (Fig. 4-E).

\section{DISCUSSION}

In the present work, the mortality rate among the DOX-treated rats was very high; as 5 of 12 rats died in the DOX group and 7 of 12 rats died in the withdrawal group. Previous studies ${ }^{4,16,17,18}$ were in agreement with these results and they attributed these findings to the development of cardiomyopathy and deterioration of cardiac function after DOX treatment.

These results were in contrary to Razmaraii et al. ${ }^{19}$ who noted that there was no significant change in the mortality rate between DOX-treated rats and the rats in the control, protective and withdrawal groups.

In the present study, the mortality rate was significantly declined by concomitant use of metformin with DOX (8\% mortality only). Also, there was no mortality in the control and metformin groups. These results were also observed by previous studies ${ }^{11,13,14,20}$.

Regarding BW, HW, and HW/BW ratio, there was a highly significant difference between the studied groups. Also, LSD test comparing between the studied groups showed that the values of weight and ratio in DOX and withdrawal groups were significantly decreased compared to other groups. These results were observed previously by other studies ${ }^{4,16}$. They attributed these findings to decreased appetite in DOX-treated rats and inhibition of protein production caused by DOX.

In contrary to the results of this study, other studies 17,18 showed no significant difference between the studied groups.

Co-administration of metformin with DOX made the rats gain weight but still less than the weight gain in the rats in the control and metformin groups indicating that metformin decreased the toxic effect of DOX. These results were in accordance with previous studies ${ }^{13,14,20}$.

In disagreement with these results, the observations obtained by previous studies ${ }^{21,22}$ who mentioned that the rats in the combined DOX and metformin group gained weight normally typically like the rats in the control and metformin group.

Regarding the serum cardiac troponin I (cTn I), this study revealed that, there was a significant elevation of CTn I in the DOX group denoting high cardiomyocyte breakdown. The values decreased, but still higher than normal, in the withdrawal group. These values descent to just little above the normal range in the combined DOX and MET group. These results were in accordance with previous studies $4,14,18$

Like cTn I, was the results of serum CK-MB. This study revealed more elevated CK-MB levels in DOX-treated group than in withdrawal group and both of them were significantly higher than the levels in combined DOX and metformin group which was little above the normal range. The same results were observed by other studies ${ }^{4,11,13,18,20}$.

A slightly different results were obtained by previous studies ${ }^{21,22}$ as they found that the serum CTn I and serum CK-MB was returned completely to normal values in the combined DOX and metformin group. Histopathological examination of myocardium in $\mathrm{H}$ \& E stained sections from DOX-treated group showed disorganized cardiac muscle fibers and areas of wavy myocardium with intramyocardial necrosis in the form of focal cardiomyocyte necrosis or contraction band necrosis (necrosis of adjacent cardiomyocytes). There were also ill-defined or lost cell borders, pyknotic or absent nuclei, pale eosinophilic cytoplasm and scattered inflammatory cells. Furthermore, there were scattered vacuoles, areas of hemorrhage, and other areas of congestion showing numerous RBCs in between cardiomyocytes. These observations were nearly the same in the withdrawal group, but less aggressive and also occupied a lesser area of the myocardium in comparison with DOX group. These findings were mentioned by other studies $4,11,13,14,17$.

Moreover, in accordance with other studies $4,11,22$, Masson's trichrome stained sections in the DOXtreated group showed a very thick irregular collagen fibers in between the cardiomyocytes. This was completely different from the control and metformin groups which showed a very thin or nearly absent collagen fibers in between the cardiomyocytes.

This study also demonstrated that, $\mathrm{H}$ \& $\mathrm{E}$ stained sections from the combined DOX and metformin group showed a significant improvement in the above-mentioned toxic effects in comparison with DOX and withdrawal groups; as the myocardium appeared well-organized with no hemorrhagic nor congested areas. Most of cardiomyocytes appeared normal nuclei and cytoplasm with little areas of wavy myocardium. These observations denote that metformin has a great protective effect on DOXinduced cardiotoxicity and were in agreement with previous studies ${ }^{11,13,14,20}$.

Furthermore, Masson's trichrome stained sections in the combined DOX and metformin group showed a very thin or nearly absent collagen fibers in between cardiomyocytes like the collagen distribution in the control and metformin groups, denoting that metformin prevented DOX-induced myocardial fibrosis. This was in accordance with previous studies ${ }^{11,22}$.

Regarding ultra-structural changes, TEM results of DOX-treated group showed focal areas of myofibrillar loss with disorganized A and I bands. The majority of nuclei were pyknotic with aggregated chromatin in some parts with wide areas of chromatin loss in other parts. Peri-nuclear mitochondria were nearly absent around the pyknotic nuclei. Most IFM appeared fragmented with disrupted cristae. Wide, separated, interrupted intercalated discs were noted between the cardiac myocytes. The same observations were found in withdrawal group, but were less severe than in the DOX group. This was in agreement with previous studies $4,20,23,24$. 
This study also demonstrated that, concomitant use of metformin with DOX ameliorated the ultrastructural changes caused by DOX. TEM results of this group showed a picture nearly similar to the control and metformin groups; as the majority of cardiomyocytes had well-organized configuration with minor loss in limited areas. Also, the nuclei and mitochondria were normal in appearance except many alterations in some parts. These were in accordance with Ajmal et al. ${ }^{20}$ who attributed these changes to the cardioprotective effect of MET on the mitochondrial level mainly and also on the nuclear and DNA level.

\section{CONCLUSION}

These results proved histologically and biochemically that metformin has an evident protective effect on myocardial injury induced by doxorubicin. Therefore, concomitant use of metformin with doxorubicin is suggested in cancer patients.

\section{REFERENCES}

1. Bray F, Ferlay J, Soerjomataram I, et al. Global cancer statistics 2018: GLOBOCAN Estimates of Incidence and Mortality Worldwide for 36 Cancers in 185 Countries. CA: A Cancer Journal for Clinicians. 2018; 68 (6): 394-424.

2. McGowan JV, Chung R, Maulik A, et al. Anthracycline chemotherapy and cardiotoxicity. Cardiovascular drugs and therapy. 2017; 31 (1): 63-75.

3. McKenna WJ, Maron BJ and Thiene G. Classification, epidemiology, and global burden of cardiomyopathies. Circulation Research. 2017; 121 (7): 722-30.

4. Dundar HA, Kiray M, Kir M, et al. Protective effect of acetyl-L-carnitine against doxorubicininduced cardiotoxicity in Wistar albino rats. Archives of Medical Research. 2016; 47 (7): 506-14.

5. Renu K, Abilash VG, Pb TP, et al. Molecular mechanism of doxorubicin-induced cardiomyopathy-An update. European Journal of Pharmacology. 2018; 818 (1): 241-53.

6. Prathumsap N, Shinlapawittayatorn K, Chattipakorn SC, et al. Effects of doxorubicin on the heart: From molecular mechanisms to intervention strategies. European Journal of Pharmacology. 2019; 866 (1): 1-17.

7. Wenningmann $\mathrm{N}$, Knapp $\mathrm{M}$, Ande $\mathrm{A}$, et al. Insights into doxorubicin-induced cardiotoxicity: molecular mechanisms, preventive strategies, and early monitoring. Molecular Pharmacology. 2019; 96 (2): 219-32.

8. Chung WB and Youn HJ. Pathophysiology and preventive strategies of anthracycline-induced cardiotoxicity. The Korean Journal of Internal Medicine. 2016; 31 (4): 625-33.

9. Driver C, Bamitale KD, Kazi A, et al. Cardioprotective effects of metformin. Journal of Cardiovascular Pharmacology. 2018; 72 (2): 121-7.

10. American Diabetes Association. Pharmacologic Approaches to Glycemic Treatment: Standards of Medical Care in Diabetes-2021. Diabetes Care. 2021; 44 (1): S111-24.
11. Zilinyi R, Czompa A, Czegledi A, et al. The cardioprotective effect of metformin in doxorubicin-induced cardiotoxicity: the role of autophagy. Molecules. 2018; 23 (5): 1184-95.

12. Oh TK and Song IA. Metformin Use and the Risk of Cancer in Patients with Diabetes: A Nationwide Sample Cohort Study. Cancer Prevention Research. 2020; 13 (2): 195-202.

13. Argun $M$, Üzüm $K$, Sönmez $M F$, et al. Cardioprotective effect of metformin against doxorubicin cardiotoxicity in rats. Anatolian Journal of Cardiology. 2016; 16 (4): 234-41.

14. Chen J, Zhang S, Pan G, et al. Modulatory effect of metformin on cardiotoxicity induced by doxorubicin via the MAPK and AMPK pathways. Life Sciences. 2020; 249 (1): 1-11.

15. Suvarna SK, Layton $C$ and Bancroft JD. Bancroft's theory and practice of histological techniques, 8th ed, Elsevier, London, Edinburgh, New York, Philadelphia, St Louis, Sydney and Toronto; 2019. PP. 40-61, 126-37, 165, 66 and 434-75.

16. Abe J, Yamada Y, Takeda A, et al. Cardiac progenitor cells activated by mitochondrial delivery of resveratrol enhance the survival of a doxorubicin-induced cardiomyopathy mouse model via the mitochondrial activation of a damaged myocardium. Journal of Controlled Release. 2018; 269 (1): 177-88.

17. Agustini FD, Arozal W, Louisa $M$, et al. Cardioprotection mechanism of mangiferin on doxorubicin-induced rats: focus on intracellular calcium regulation. Pharmaceutical Biology. 2016; 54 (7): 1289-97.

18. Bartlett JJ, Trivedi PC, Yeung $P$, et al. Doxorubicin impairs cardiomyocyte viability by suppressing transcription factor EB expression and disrupting autophagy. Biochemical Journal. 2016; 473 (21): 3769-89.

19. Razmaraii N, Babaei H, Nayebi AM, et al. Cardioprotective effect of phenytoin on doxorubicin-induced cardiac toxicity in a rat model. Journal of Cardiovascular Pharmacology. 2016; 67 (3): 237-45.

20. Ajmal K, Rafique $S$, Afzal A, et al. Protective effects of metformin on doxorubicin-induced cardiotoxicity and its early detection. Medical Forum. 2020; 31 (9): 185-9.

21. Mackay $\mathrm{AD}$, Marchant ED, Munk DJ, et al. Multitissue analysis of exercise and metformin on doxorubicin-induced iron dysregulation. American Journal of Physiology-Endocrinology and Metabolism. 2019; 316 (5): E922-30.

22. Shaty MH, Arif IS, Al-Ezzi MI, et al. Metformin attenuate fibrosis in both acute and chronic doxorubicin cardiotoxicity in rabbits. Journal of Pharmaceutical Sciences and Research. 2018; 10 (6): 1559-65.

23. Botelho AFM, Lempek MR, Branco SEM, et al. Coenzyme Q10 cardioprotective effects against doxorubicin-induced cardiotoxicity in Wistar Rat. Cardiovascular Toxicology. 2019; 20: 22234.

24. Zhang YY, Yi M and Huang YP. Oxymatrine ameliorates doxorubicin-induced cardiotoxicity in rats. Cellular Physiology and Biochemistry. 2017; 43 (2): 626-35. 